\title{
Existence theory to a class of boundary value problems of hybrid fractional sequential integro-differential equations
}

\author{
M. Jamil ${ }^{1 *}$ D, R.A. Khan ${ }^{1}$ and K. Shah'
}

"Correspondence:

jamilkanju@gmail.com

${ }^{1}$ Department of Mathematics,

University of Malakand, Chakdara

Dir(Lower), khyber Pakhtunkhwa,

Pakistan

\begin{abstract}
In this article, we study the existence result for a boundary value problem (BVP) of hybrid fractional sequential integro-differential equations. A fixed point theorem provided by Dhage in (Nonlinear Anal. 4:414-424, 2010) is used for the solution existence of our boundary value problem. Also we illustrated our result through an example.
\end{abstract}

MSC: Primary 26A33; 34A08; secondary 34A38

Keywords: Boundary value problem; Hybrid fractional sequential integro-differential equation; Fixed point theorem

\section{Introduction}

Fractional calculus is a branch of mathematics which investigates the properties of integrals and derivatives of non-integer order. These integrals and derivatives of non-integer order are called fractional integrals and derivatives. The potential of fractional calculus has drawn the attention of applied scientists, engineers, and other researchers. Also theoretical scientists or researchers are interested in the field of fractional derivatives and fractional integration. Chronologically, in [2] we can find different approaches of fractional calculus proposed by Liouville, Euler, Fourier, Riemann, Abel, etc. We can find some relevant work on fractional calculus in a series of papers [3-28].

In the area of fractional calculus, several researchers have studied hybrid fractional differential equations, see [1,29-32] and the references therein. Recently in [33], the authors developed an existence result for a boundary value problem of hybrid fractional differential equations of the form

$$
\left\{\begin{array}{l}
D^{\alpha}\left(\frac{\phi(t)-\psi(t, \phi(t))}{\theta(t, \phi(t))}\right)=\varphi(t, \phi(t)), \quad t \in[0,1], \alpha \in(1,2], \\
{\left.\left[\frac{\phi(t)-\psi(t, \phi(t))}{\theta(t, \phi(t))}\right]\right|_{t=0}=0,\left.\quad\left[\frac{\phi(t)-\psi(t, \phi(t))}{\theta(t, \phi(t))}\right]\right|_{t=1}=0,}
\end{array}\right.
$$

where $\theta \in C(\mathbb{J} \times \mathbb{R}, \mathbb{R} \backslash\{0\}), \psi, \varphi \in C(\mathbb{J} \times \mathbb{R}, \mathbb{R})$. 
In [34], Sitho derived an existence result for an initial value problem of hybrid fractional sequential integro-differential equations of the form

$$
\left\{\begin{array}{l}
D^{\alpha}\left[\frac{D^{\omega} u(t)-\sum_{i=1}^{m} I^{\beta_{i}} f_{i}(t, u(t))}{g(t, u(t))}\right]=h\left(t, u(t), I^{\gamma} u(t)\right), \quad t \in[0, T] \\
u(0)=0, \quad D^{\omega} u(0)=0
\end{array}\right.
$$

where $D^{\alpha}, D^{\omega}$ denote the Riemann-Liouville fractional derivatives of order $\alpha, \omega$ respectively and $0<\alpha, \omega \leq 1$.

In this article, we study the existence result for the boundary value problem of hybrid fractional sequential integro-differential equations involving Caputo derivatives given by

$$
\begin{cases}{ }^{c} D^{\alpha}\left[\frac{{ }^{c} D^{\omega} u(t)-\sum_{i=1}^{m} I^{\beta} f_{i}(t, u(t))}{g(t, u(t))}\right]=h\left(t, u(t), I^{\gamma} u(t)\right), & t \in \mathbb{J}=[0,1], \\ u(0)=0, \quad D^{\omega} u(0)=0, \quad u(1)=\delta u(\eta), & 0<\delta<1,0<\eta<1,\end{cases}
$$

where ${ }^{c} D^{\alpha}$ is the Caputo fractional derivative of order $\alpha,{ }^{c} D^{\omega}$ is the Caputo fractional derivative of order $\omega, 0<\alpha \leq 1,1<\omega \leq 2, I^{\gamma}$ denotes the Riemann-Liouville fractional integral of order $\gamma>0, I^{\beta_{i}}$ denotes the Riemann-Liouville fractional sequential integrals of order $\beta_{i}>0, g \in C(\mathbb{J} \times \mathbb{R}, \mathbb{R} \backslash\{0\}), h \in C\left(\mathbb{J} \times \mathbb{R}^{2}, \mathbb{R}\right)$ and $f_{i} \in C(\mathbb{J} \times \mathbb{R}, \mathbb{R})$ with $f_{i}(0,0)=0$, $i=1,2, \ldots, m$.

In comparison to problem (1), our considered BVP (2) is more general than the problem studied in [34], as we consider a problem with three point boundary conditions, while the authors in [34] investigated an initial value problem. Moreover, in our problem the fractional orders of derivatives are $0<\alpha \leq 1$ and $1<\omega \leq 2$, whereas in problem (1) the fractional orders are $0<\alpha, \omega \leq 1$.

We obtain an existence result for the boundary value problem (2) in Sect. 3 by using generalized Krasnoselskii's fixed point theorem provided by Dhage in [1].

\section{Preliminaries}

This section provides some important definitions of fractional calculus $[2,35,36]$ and results of fixed point theory $[1,20,21]$, which is base for the forthcoming sections.

Definition 1 The Caputo fractional derivative of positive real order $\omega>0$ of a function $u(t)$ is given by

$$
{ }^{c} D^{\omega} u(t)=\frac{1}{\Gamma(n-\omega)} \int_{0}^{t} \frac{u^{(n)}(s)}{(t-s)^{\omega-n+1}} d s, \quad \omega>0
$$

provided that the integral on the right-hand side exists.

Definition 2 The Riemann-Liouville fractional derivative of positive real order $\omega>0$ of a function $u(t)$ is given by

$$
D^{\omega} u(t)=\frac{1}{\Gamma(n-\omega)} \frac{d^{n}}{d t^{n}} \int_{0}^{t} \frac{u(s)}{(t-s)^{\omega-n+1}} d s, \quad \omega>0
$$

provided that the integral on the right-hand side exists. 
Definition 3 The Riemann-Liouville fractional integral of positive real order $\omega>0$ of a function $u(t)$ is given by

$$
I^{\omega} u(t)=\frac{1}{\Gamma(\omega)} \int_{0}^{t}(t-s)^{\omega-1} u(s) d s, \quad \omega>0,
$$

provided that the integral on the right-hand side exists.

Remark 1 For Definitions $1-3, n=[\omega]+1,[\omega]$ is the greatest integer less than $\omega$ and $\Gamma$ is the gamma function defined by $\Gamma(\omega)=\int_{0}^{\infty} e^{-s} s^{\omega-1} d s$.

Property 1 ([36]) Let $\beta, \omega>0$ and $u(t)$ be a function, then the semi-group property for the Riemann-Liouville fractional integrals of orders $\beta$ and $\omega$ respectively is given by

$$
I^{\beta}\left[I^{\omega} u(t)\right]=I^{\beta+\omega} u(t)
$$

Next we present two important results as lemmas which we need later.

Lemma 1 ([37]) For $u \in C(0, T) \cap L(0, T)$, the solution of the Caputo fractional differential equation

$$
{ }^{c} D^{\omega} u(t)=g(t), \quad n-1<\omega<n,
$$

is given by

$$
u(t)=I^{\omega} g(t)+\sum_{i=0}^{n-1} k_{i} t^{i}
$$

or

$$
u(t)=I^{\omega} g(t)+k_{0}+k_{1} t+\cdots+k_{n-1} t
$$

where $n=[\omega]+1,[\omega]$ is the greatest integer less than $\omega$.

Let $E=C(\mathbb{J}, \mathbb{R})$ be the Banach space of continuous real-valued functions defined on $\mathbb{J}=$ $[0,1]$. We define a norm $\|\cdot\|$ and a multiplication in $E$ by $\|u\|=\sup _{t \in \mathbb{J}}|u(t)|$ and $(u v)(t)=$ $u(t) v(t), \forall t \in \mathbb{J}$. Then clearly $E$ is a Banach algebra with above defined supremum norm and multiplication in it.

Lemma 2 ([1]) Let $S$ be a nonempty, convex, closed, and bounded set such that $S \subseteq E$, and let $A: E \rightarrow E$ and $B: S \rightarrow E$ be two operators which satisfy the following:

$\left(c_{1}\right) A$ is contraction,

$\left(c_{2}\right) B$ is compact, and

(c) $u=A u+B v \forall v \in S \Longrightarrow u \in S$.

Then there exists a solution of the operator equation $u=A u+B u$. 


\section{Solution existence of hybrid fractional sequential integro-differential equations}

In this section, we consider the hybrid fractional sequential integro-differential equation boundary value problem (2). First we derive its solution and then discuss the existence of solution using the above stated definitions and results of the preliminary section.

Lemma 3 Suppose that $0<\alpha \leq 1,1<\omega \leq 2, \gamma>0$, and functions $g, h, f_{i}, i=1,2, \ldots, m$, satisfy $B V P(2)$. Then the unique solution of $B V P(2)$ is given by

$$
\begin{aligned}
u(t)= & \int_{0}^{t} \frac{(t-s)^{\omega-1}}{\Gamma(\omega)} g(s, u(s)) \int_{0}^{s} \frac{(s-\mu)^{\alpha-1}}{\Gamma(\alpha)} h\left(\mu, u(\mu), I^{\gamma} u(\mu)\right) d \mu d s \\
& +\sum_{i=1}^{m} I^{\beta_{i}+\omega} f_{i}(t, u(t)) \\
& +\frac{t}{\delta \eta-1}\left[\int_{0}^{1} \frac{(1-s)^{\omega-1}}{\Gamma(\omega)} g(s, u(s)) \int_{0}^{s} \frac{(s-\mu)^{\omega-1}}{\Gamma(\alpha)} h\left(\mu, u(\mu), I^{\gamma} u(\mu)\right) d \mu d s\right. \\
& +\sum_{i=1}^{m} I^{\beta_{i}+\omega} f_{i}(1, u(1)) \\
& -\delta\left(\int_{0}^{\eta} \frac{(\eta-s)^{\omega-1}}{\Gamma(\omega)} g(s, u(s)) \int_{0}^{s} \frac{(s-\mu)^{\omega-1}}{\Gamma(\alpha)} h\left(\mu, u(\mu), I^{\gamma} u(\mu)\right) d \mu d s\right. \\
& \left.\left.+\sum_{i=1}^{m} I^{\beta_{i}+\omega} f_{i}(\eta, u(\eta))\right)\right],
\end{aligned}
$$

where

$$
\begin{aligned}
& I^{\beta_{i}+\omega} f_{i}(t, u(t))=\int_{0}^{t} \frac{(t-s)^{\beta_{i}+\omega-1}}{\Gamma\left(\beta_{i}+\omega\right)} f_{i}(s, u(s)) d s, \\
& I^{\beta_{i}+\omega} f_{i}(1, u(1))=\int_{0}^{1} \frac{(1-s)^{\beta_{i}+\omega-1}}{\Gamma\left(\beta_{i}+\omega\right)} f_{i}(s, u(s)) d s,
\end{aligned}
$$

and

$$
I^{\beta_{i}+\omega} f_{i}(\eta, u(\eta))=\int_{0}^{\eta} \frac{(\eta-s)^{\beta_{i}+\omega-1}}{\Gamma\left(\beta_{i}+\omega\right)} f_{i}(s, u(s)) d s .
$$

Proof Assigning the Riemann-Liouville fractional integral operator of order $\alpha$ to hybrid fractional sequential integro-differential equations (2) and using Lemma 1, we have

$$
\frac{{ }^{c} D^{\omega} u(t)-\sum_{i=1}^{m} I^{\beta_{i}} f_{i}(t, u(t))}{g(t, u(t))}=I^{\alpha} h\left(t, u(t), I^{\gamma} u(t)\right)+k_{0} .
$$

In view of the initial conditions $u(0)=0, D^{\omega} u(0)=0$ of problem (2) with $f_{i}(0,0)=0$, we have $k_{0}=0$, and then equation (4) takes the form

$$
{ }^{c} D^{\omega} u(t)=g(t, u(t)) \int_{0}^{t} \frac{(t-s)^{\alpha-1}}{\Gamma(\alpha)} h\left(s, u(s), I^{\gamma} u(s)\right) d s+\sum_{i=1}^{m} I^{\beta_{i}} f_{i}(t, u(t))
$$


Now assigning the Riemann-Liouville fractional integral operator of order $\omega$ to equation (5) and using Lemma 1 with semi-group Property 1, we have

$$
\begin{aligned}
u(t)= & \int_{0}^{t} \frac{(t-s)^{\omega-1}}{\Gamma(\omega)} g(s, u(s)) \int_{0}^{s} \frac{(s-\mu)^{\alpha-1}}{\Gamma(\alpha)} h\left(\mu, u(\mu), I^{\gamma} u(\mu)\right) d \mu d s \\
& +\sum_{i=1}^{m} I^{\beta_{i}+\omega} f_{i}(t, u(t))+k_{1}+k_{2} t .
\end{aligned}
$$

The initial condition $u(0)=0$ of problem (2) gives $k_{1}=0$, for which equation (6) becomes

$$
\begin{aligned}
u(t)= & \int_{0}^{t} \frac{(t-s)^{\omega-1}}{\Gamma(\omega)} g(s, u(s)) \int_{0}^{s} \frac{(s-\mu)^{\alpha-1}}{\Gamma(\alpha)} h\left(\mu, u(\mu), I^{\gamma} u(\mu)\right) d \mu d s \\
& +\sum_{i=1}^{m} I^{\beta_{i}+\omega} f_{i}(t, u(t))+k_{2} t
\end{aligned}
$$

and then the boundary condition $u(1)=\delta u(\eta)$ of problem (2) gives

$$
\begin{aligned}
k_{2}= & \frac{1}{\delta \eta-1}\left[\int_{0}^{1} \frac{(1-s)^{\omega-1}}{\Gamma(\omega)} g(s, u(s)) \int_{0}^{s} \frac{(s-\mu)^{\omega-1}}{\Gamma(\alpha)} h\left(\mu, u(\mu), I^{\gamma} u(\mu)\right) d \mu d s\right. \\
& +\sum_{i=1}^{m} I^{\beta_{i}+\omega} f_{i}(1, u(1)) \\
& -\delta\left(\int_{0}^{\eta} \frac{(\eta-s)^{\omega-1}}{\Gamma(\omega)} g(s, u(s)) \int_{0}^{s} \frac{(s-\mu)^{\omega-1}}{\Gamma(\alpha)} h\left(\mu, u(\mu), I^{\gamma} u(\mu)\right) d \mu d s\right. \\
& \left.\left.+\sum_{i=1}^{m} I^{\beta_{i}+\omega} f_{i}(\eta, u(\eta))\right)\right]
\end{aligned}
$$

for which equation (7) takes the form

$$
\begin{aligned}
u(t)= & \int_{0}^{t} \frac{(t-s)^{\omega-1}}{\Gamma(\omega)} g(s, u(s)) \int_{0}^{s} \frac{(s-\mu)^{\alpha-1}}{\Gamma(\alpha)} h\left(\mu, u(\mu), I^{\gamma} u(\mu)\right) d \mu d s \\
& +\sum_{i=1}^{m} I^{\beta_{i}+\omega} f_{i}(t, u(t)) \\
+ & \frac{t}{\delta \eta-1}\left[\int_{0}^{1} \frac{(1-s)^{\omega-1}}{\Gamma(\omega)} g(s, u(s)) \int_{0}^{s} \frac{(s-\mu)^{\omega-1}}{\Gamma(\alpha)} h\left(\mu, u(\mu), I^{\gamma} u(\mu)\right) d \mu d s\right. \\
& +\sum_{i=1}^{m} I^{\beta_{i}+\omega} f_{i}(1, u(1)) \\
- & \delta\left(\int_{0}^{\eta} \frac{(\eta-s)^{\omega-1}}{\Gamma(\omega)} g(s, u(s)) \int_{0}^{s} \frac{(s-\mu)^{\omega-1}}{\Gamma(\alpha)} h\left(\mu, u(\mu), I^{\gamma} u(\mu)\right) d \mu d s\right. \\
& \left.\left.+\sum_{i=1}^{m} I^{\beta_{i}+\omega} f_{i}(\eta, u(\eta))\right)\right] .
\end{aligned}
$$

Hence we obtain the unique solution of BVP (2). Thus the proof is completed. 
For developing the existence result, we consider some assumptions which are the following.

$\left(A_{1}\right)$ The functions $f_{i} \in C(\mathbb{J} \times \mathbb{R}, \mathbb{R}), g \in C(\mathbb{J} \times \mathbb{R}, \mathbb{R} \backslash\{0\})$, and $h \in C\left(\mathbb{J} \times \mathbb{R}^{2}, \mathbb{R}\right)$ are continuous, and there exist positive functions $\lambda_{i}(t), \Theta(t)$, and $\psi(t)$ with bounds $\left\|\lambda_{i}\right\|$, $\|\Theta\|$, and $\|\psi\|$ respectively such that

$$
\begin{aligned}
& \mid f_{i}(t, u(t))-f_{i}\left(t, v(t)\left|\leq \lambda_{i}(t)\right| u(t)-v(t) \mid\right. \\
& \mid g(t, u(t))-g(t, v(t)|\leq \Theta(t)| u(t)-v(t) \mid \\
& \text { and } \mid h(t, u(t), \bar{u}(t))-h(t, v(t), \bar{v}(t) \mid \leq \psi(t)(|u(t)-v(t)|+|\bar{u}(t)-\bar{v}(t)|)
\end{aligned}
$$

for $t \in \mathbb{J}$ and $u, v \in \mathbb{R}$.

$\left(A_{2}\right)\left|f_{i}(t, u)\right| \leq \phi_{i}(t), \forall(t, u) \in \mathbb{J} \times \mathbb{R}, \phi_{i} \in C\left(\mathbb{J}, \mathbb{R}_{+}\right), i=1,2,3, \ldots, m,|h(t, u, v)| \leq \Omega(t)$, $\forall(t, u, v) \in \mathbb{J} \times \mathbb{R} \times \mathbb{R}, \Omega \in C\left(\mathbb{J}, \mathbb{R}_{+}\right),|g(t, u)| \leq \chi(t), \forall(t, u) \in \mathbb{J} \times \mathbb{R}, \chi \in C\left(\mathbb{J}, \mathbb{R}_{+}\right)$.

$\left(A_{3}\right)$ There exists $r>0$ such that

$$
\left(1+\frac{1+\delta}{|\delta \eta-1|}\right)\left[\frac{\|\chi\|\|\Omega\|}{\Gamma(\alpha+1) \Gamma(\omega+1)}+\sum_{i=1}^{m} \frac{\left\|\phi_{i}\right\|}{\Gamma\left(\beta_{i}+\omega+1\right)}\right] \leq r
$$

and

$$
\left(1+\frac{1+\delta}{|\delta \eta-1|}\right)\left[\frac{\|\Omega\|\|\Theta\|+\|\chi\|\|\psi\|}{\Gamma(\alpha+1) \Gamma(\omega+1)}+\frac{\|\chi\|\|\psi\|}{\Gamma(\alpha+1) \Gamma(\omega+1) \Gamma(\gamma+1)}\right]<1 .
$$

Our main existence result is based on generalized Krasnoselikii's fixed point theorem by Dhage [1], which we have provided in Lemma 2.

Theorem 2 Let assumptions $\left(A_{1}\right)-\left(A_{3}\right)$ hold, then there exists at least one solution for BVP (2) in $\mathbb{J}=[0,1]$.

Proof First we set $\sup _{t \in \mathbb{J}}\left|\phi_{i}(t)\right|=\left\|\phi_{i}\right\|, \sup _{t \in \mathbb{J}}\left|\lambda_{i}(t)\right|=\left\|\lambda_{i}\right\|, i=1,2, \ldots, m, \sup _{t \in \mathbb{J}}|\Theta(t)|=$ $\|\Theta\|, \sup _{t \in \mathbb{J}}|\psi(t)|=\|\psi\|, \sup _{t \in \mathbb{J}}|\Omega(t)|=\|\Omega\|$, and $\sup _{t \in \mathbb{J}}|\chi(t)|=\|\chi\|$.

Now we consider $E=C(\mathbb{J}, \mathbb{R})$ and define $S \subseteq E$ as

$$
S=\{u \in E:\|u\| \leq r\}
$$

Clearly $S$ is a closed, convex, and bounded subset of the Banach space $E$. Let us define two operators $C: E \longrightarrow E$ and $D: E \longrightarrow E$ such that

$$
C u(t)=\int_{0}^{t} \frac{(t-s)^{\alpha-1}}{\Gamma(\alpha)} h\left(s, u(s), I^{\gamma} u(t)\right) d s
$$

and

$$
D u(t)=g(t, u(t)) .
$$

Then, using assumptions $\left(A_{1}\right),\left(A_{2}\right)$ and proceeding with maximum over $\mathbb{J}$, we have

$$
\|C u(t)-C v(t)\| \leq \frac{\|\psi\|}{\Gamma(\alpha+1)}\left(1+\frac{1}{\Gamma(\gamma+1)}\right)\|u-v\|,
$$




$$
\begin{aligned}
& \|D u(t)-D v(t)\| \leq\|\Theta\|\|u-v\|, \\
& \|C u(t)\| \leq \frac{\|\Omega\|}{\Gamma(\alpha+1)} \\
& \text { and } \quad\|D u(t)\| \leq\|\chi\| .
\end{aligned}
$$

Now we define two more operators $A: E \longrightarrow E$ and $B: S \longrightarrow E$ such that

$$
\begin{aligned}
A u(t)= & \int_{0}^{t} \frac{(t-s)^{\omega-1}}{\Gamma(\omega)} D u(s) C u(s) d s+\frac{t}{\delta \eta-1} \int_{0}^{1} \frac{(1-s)^{\omega-1}}{\Gamma(\omega)} D u(s) C u(s) d s \\
& -\frac{\delta t}{\delta \eta-1} \int_{0}^{\eta} \frac{(\eta-s)^{\omega-1}}{\Gamma(\omega)} D u(s) C u(s) d s, \quad t \in \mathbb{J}
\end{aligned}
$$

and

$$
\begin{aligned}
B u(t)= & \sum_{i=1}^{m} I^{\beta_{i}+\omega} f_{i}(t, u(t))+\frac{t}{\delta \eta-1} \sum_{i=1}^{m} I^{\beta_{i}+\omega} f_{i}(1, u(1)) \\
& -\frac{\delta t}{\delta \eta-1} \sum_{i=1}^{m} I^{\beta_{i}+\omega} f_{i}(\eta, u(\eta)), \quad t \in \mathbb{J},
\end{aligned}
$$

or

$$
\begin{aligned}
B u(t)= & \sum_{i=1}^{m} \int_{0}^{t} \frac{(t-s)^{\beta_{i}+\omega-1}}{\Gamma\left(\beta_{i}+\omega\right)} f_{i}(s, u(s)) d s+\frac{t}{\delta \eta-1} \sum_{i=1}^{m} \int_{0}^{1} \frac{(1-s)^{\beta_{i}+\omega-1}}{\Gamma\left(\beta_{i}+\omega\right)} f_{i}(s, u(s)) d s \\
& -\frac{\delta t}{\delta \eta-1} \sum_{i=1}^{m} \int_{0}^{\eta} \frac{(\eta-s)^{\beta_{i}+\omega-1}}{\Gamma\left(\beta_{i}+\omega\right)} f_{i}(s, u(s)) d s, \quad t \in J .
\end{aligned}
$$

Then the integral equation (3) can be written in the operator form as follows:

$$
u(t)=A u(t)+B u(t), \quad t \in J .
$$

We show that the two operators $A$ and $B$ in (15) satisfy all the conditions of Lemma 2 . This can be achieved in the following steps.

Step 1. First we show that $A$ is a contraction mapping. Let $u(t), v(t) \in S$, then we have

$$
\begin{aligned}
\mid A u(t) & -A v(t) \mid \\
\leq & \int_{0}^{t} \frac{(t-s)^{\omega-1}}{\Gamma(\omega)}|D u(s) C u(s)-D v(s) C v(s)| d s \\
& +\frac{t}{|\delta \eta-1|} \int_{0}^{1} \frac{(1-s)^{\omega-1}}{\Gamma(\omega)}|D u(s) C u(s)-D v(s) C v(s)| d s \\
& +\frac{\delta t}{|\delta \eta-1|} \int_{0}^{\eta} \frac{(\eta-s)^{\omega-1}}{\Gamma(\omega)}|D u(s) C u(s)-D v(s) C v(s)| d s \\
\leq & \left(1+\frac{1}{|\delta \eta-1|}+\frac{\delta}{|\delta \eta-1|}\right) \int_{0}^{1} \frac{(1-s)^{\omega-1}}{\Gamma(\omega)}|D u(s) C u(s)-D v(s) C v(s)| d s \\
\leq & \left(1+\frac{1+\delta}{|\delta \eta-1|}\right)\left[\int_{0}^{1} \frac{(1-s)^{\omega-1}}{\Gamma(\omega)} \mid D u(s) C u(s)-C u(s) D v(s)\right.
\end{aligned}
$$




$$
\begin{aligned}
& +C u(s) D v(s)-D v(s) C v(s) \mid d s] \\
\leq & \left(1+\frac{1+\delta}{|\delta \eta-1|}\right)\left[\int_{0}^{1} \frac{(1-s)^{\omega-1}}{\Gamma(\omega)}|C u(s)||D u(s)-D v(s)| d s\right. \\
& \left.+\int_{0}^{1} \frac{(1-s)^{\omega-1}}{\Gamma(\omega)}|D v(s)||C u(s)-C v(s)| d s\right]
\end{aligned}
$$

which gives

$$
\begin{aligned}
\|A u(t)-A v(t)\| \leq & \left(1+\frac{1+\delta}{|\delta \eta-1|}\right)\left[\frac{\|\Omega\|\|\Theta\|+\|\chi\|\|\psi\|}{\Gamma(\alpha+1) \Gamma(\omega+1)}\right. \\
& \left.+\frac{\|\chi\|\|\psi\|}{\Gamma(\alpha+1) \Gamma(\omega+1) \Gamma(\gamma+1)}\right]\|u-v\| .
\end{aligned}
$$

Hence by (8) the operator $A$ is a contraction mapping.

Step 2. Next we show that the operator $B$ satisfies condition $\left(c_{2}\right)$ of Lemma 2, that is, the operator $B$ is compact on $S$. Therefore first we show that the operator $B$ is continuous on $S$. Let $\left\{u_{n}(t)\right\}$ be a sequence of functions in $S$ converging to a function $u(t) \in S$. Then, by the Lebesgue dominant convergence theorem, $\forall t \in \mathbb{J}$, we have

$$
\begin{aligned}
\lim _{n \rightarrow \infty} B u_{n}(t)= & \lim _{n \rightarrow \infty}\left[\sum_{i=1}^{m} \int_{0}^{t} \frac{(t-s)^{\beta_{i}+\omega-1}}{\Gamma\left(\beta_{i}+\omega\right)} f_{i}\left(s, u_{n}(s)\right) d s\right. \\
& +\frac{t}{\delta \eta-1} \sum_{i=1}^{m} \int_{0}^{1} \frac{(1-s)^{\beta_{i}+\omega-1}}{\Gamma\left(\beta_{i}+\omega\right)} f_{i}\left(s, u_{n}(s)\right) d s \\
& \left.-\frac{\delta t}{\delta \eta-1} \sum_{i=1}^{m} \int_{0}^{\eta} \frac{(\eta-s)^{\beta_{i}+\omega-1}}{\Gamma\left(\beta_{i}+\omega\right)} f_{i}\left(s, u_{n}(s)\right) d s\right] \\
= & \sum_{i=1}^{m} \int_{0}^{t} \frac{(t-s)^{\beta_{i}+\omega-1}}{\Gamma\left(\beta_{i}+\omega\right)} \lim _{n \rightarrow \infty} f_{i}\left(s, u_{n}(s)\right) d s \\
& +\frac{t}{\delta \eta-1} \sum_{i=1}^{m} \int_{0}^{1} \frac{(1-s)^{\beta_{i}+\omega-1}}{\Gamma\left(\beta_{i}+\omega\right)} \lim _{n \rightarrow \infty} f_{i}\left(s, u_{n}(s)\right) d s \\
& -\frac{\delta t}{\delta \eta-1} \sum_{i=1}^{m} \int_{0}^{\eta} \frac{(\eta-s)^{\beta_{i}+\omega-1}}{\Gamma\left(\beta_{i}+\omega\right)} \lim _{n \rightarrow \infty} f_{i}\left(s, u_{n}(s)\right) d s \\
= & \sum_{i=1}^{m} \int_{0}^{t} \frac{(t-s)^{\beta_{i}+\omega-1}}{\Gamma\left(\beta_{i}+\omega\right)} f_{i}(s, u(s)) d s \\
& +\frac{t}{\delta \eta-1} \sum_{i=1}^{m} \int_{0}^{1} \frac{(1-s)^{\beta_{i}+\omega-1}}{\Gamma\left(\beta_{i}+\omega\right)} f_{i}(s, u(s)) d s \\
& -\frac{\delta t}{\delta \eta-1} \sum_{i=1}^{m} \int_{0}^{\eta} \frac{(\eta-s)^{\beta_{i}+\omega-1}}{\Gamma\left(\beta_{i}+\omega\right)} f_{i}(s, u(s)) d s .
\end{aligned}
$$

Hence $\lim _{n \rightarrow \infty} B u_{n}(t)=B u(t)$. Thus $B$ is a continuous operator on $S$. Further, we show that the operator $B$ is uniformly bounded on $S$. For any $u \in S$, we have

$$
|B u(t)| \leq \sum_{i=1}^{m} \int_{0}^{t} \frac{(t-s)^{\beta_{i}+\omega-1}}{\Gamma\left(\beta_{i}+\omega\right)}\left|f_{i}(s, u(s))\right| d s
$$




$$
\begin{aligned}
& +\frac{t}{|\delta \eta-1|} \sum_{i=1}^{m} \int_{0}^{1} \frac{(1-s)^{\beta_{i}+\omega-1}}{\Gamma\left(\beta_{i}+\omega\right)}\left|f_{i}(s, u(s))\right| d s \\
& +\frac{\delta t}{|\delta \eta-1|} \sum_{i=1}^{m} \int_{0}^{\eta} \frac{(\eta-s)^{\beta_{i}+\omega-1}}{\Gamma\left(\beta_{i}+\omega\right)}\left|f_{i}(s, u(s))\right| d s \\
& \leq\left(1+\frac{1+\delta}{|\delta \eta-1|}\right) \sum_{i=1}^{m} \frac{\left\|\phi_{i}\right\|}{\Gamma\left(\beta_{i}+\omega+1\right)}:=M .
\end{aligned}
$$

Therefore $\|B u(t)\| \leq M, \forall t \in \mathbb{J}$, which shows that $B$ is uniformly bounded on $S$. Now, we show that the operator $B$ is equi-continuous. Let $t_{1}, t_{2} \in \mathbb{J}$ with $t_{1}<t_{2}$ and $u(t) \in S$. Then we have

$$
\begin{aligned}
\left|B u\left(t_{2}\right)-B u\left(t_{1}\right)\right| \leq & \mid \sum_{i=1}^{m} \int_{0}^{t_{2}} \frac{\left(t_{2}-s\right)^{\beta_{i}+\omega-1}}{\Gamma\left(\beta_{i}+\omega\right)} f_{i}(s, u(s)) d s \\
& -\sum_{i=1}^{m} \int_{0}^{t_{1}} \frac{\left(t_{1}-s\right)^{\beta_{i}+\omega-1}}{\Gamma\left(\beta_{i}+\omega\right)} f_{i}(s, u(s)) d s \\
& +\frac{t_{2}-t_{1}}{\delta \eta-1} \sum_{i=1}^{m} \int_{0}^{1} \frac{(1-s)^{\beta_{i}+\omega-1}}{\Gamma\left(\beta_{i}+\omega\right)} f_{i}(s, u(s)) d s \\
& -\frac{\delta\left(t_{2}-t_{1}\right)}{\delta \eta-1} \sum_{i=1}^{m} \int_{0}^{\eta} \frac{(\eta-s)^{\beta_{i}+\omega-1}}{\Gamma\left(\beta_{i}+\omega\right)} f_{i}(s, u(s)) d s \mid \\
\leq & \sum_{i=1}^{m} \frac{\left\|\phi_{i}\right\|}{\Gamma\left(\beta_{i}+\omega\right)} \mid \int_{0}^{t_{1}}\left[\left(t_{2}-s\right)^{\beta_{i}+\omega-1}-\left(t_{1}-s\right)^{\beta_{i}+\omega-1}\right] d s \\
& +\int_{t_{1}}^{t_{2}}\left(t_{2}-s\right)^{\beta_{i}+\omega-1} d s+\frac{t_{2}-t_{1}}{\delta \eta-1} \sum_{i=1}^{m} \int_{0}^{1}(1-s)^{\beta_{i}+\omega-1} d s \\
& -\frac{\delta\left(t_{2}-t_{1}\right)}{\delta \eta-1} \sum_{i=1}^{m} \int_{0}^{\eta}(\eta-s)^{\beta_{i}+\omega-1} d s \mid \\
\leq & \sum_{i=1}^{m} \frac{\left\|\phi_{i}\right\|}{\Gamma\left(\beta_{i}+\omega+1\right)} \mid t_{2}^{\beta_{i}+\omega}-t_{1}^{\beta_{i}+\omega}+\left(t_{2}-t_{1}\right)^{\beta_{i}+\omega} \\
& +\frac{t_{2}-t_{1}}{\delta \eta-1}-\frac{\delta \eta^{\beta_{i}+\omega}\left(t_{2}-t_{1}\right)}{\delta \eta-1} \mid .
\end{aligned}
$$

Now as $t_{2}-t_{1} \longrightarrow 0$, so the right-hand side tends to zero. Thus $B$ is equi-continuous. Therefore, it follows from the Arzelá-Ascoli theorem that $B$ is a compact operator on $S$.

Step 3. Condition $\left(c_{3}\right)$ of Lemma 2 holds. So, for any $v \in S$, we have

$$
\begin{aligned}
|u(t)|= & |A u(t)+B v(t)| \\
\leq & |A u(t)|+|B v(t)| \\
\leq & \mid \int_{0}^{t} \frac{(t-s)^{\omega-1}}{\Gamma(\omega)} D u(s) C u(s) d s+\frac{t}{\delta \eta-1} \int_{0}^{1} \frac{(1-s)^{\omega-1}}{\Gamma(\omega)} D u(s) C u(s) d s \\
& -\frac{\delta t}{\delta \eta-1} \int_{0}^{\eta} \frac{(\eta-s)^{\omega-1}}{\Gamma(\omega)} D u(s) C u(s) d s|+| \sum_{i=1}^{m} \int_{0}^{t} \frac{(t-s)^{\beta_{i}+\omega-1}}{\Gamma\left(\beta_{i}+\omega\right)} f_{i}(s, v(s)) d s
\end{aligned}
$$




$$
\begin{aligned}
& +\frac{t}{\delta \eta-1} \sum_{i=1}^{m} \int_{0}^{1} \frac{(1-s)^{\beta_{i}+\omega-1}}{\Gamma\left(\beta_{i}+\omega\right)} f_{i}(s, \nu(s)) d s \\
& -\frac{\delta t}{\delta \eta-1} \sum_{i=1}^{m} \int_{0}^{\eta} \frac{(\eta-s)^{\beta_{i}+\omega-1}}{\Gamma\left(\beta_{i}+\omega\right)} f_{i}(s, v(s)) d s \mid \\
\leq & \left(1+\frac{1+\delta}{|\delta \eta-1|}\right)\left[\frac{\|\chi\|\|\Omega\|}{\Gamma(\alpha+1) \Gamma(\omega+1)}+\sum_{i=1}^{m} \frac{\left\|\phi_{i}\right\|}{\Gamma\left(\beta_{i}+\omega+1\right)}\right] \leq r,
\end{aligned}
$$

which implies $\|u\| \leq r$, and so $u \in S$. Hence all the conditions of Lemma 2 are satisfied. Therefore, the operator equation $u(t)=A u(t)+B u(t)$ has at least one solution in $S$. Consequently, there exists a solution of $\operatorname{BVP}(2)$ in $\mathbb{J}=[0,1]$. Thus the proof is completed.

\section{Example}

We present an example of the BVP of hybrid fractional sequential integro-differential equations to test our main result.

\section{Example 1}

$$
\left\{\begin{array}{l}
{ }^{c} D^{\frac{3}{5}}\left[\frac{{ }^{c} D^{\frac{3}{2}} u(t)-\sum_{i=1}^{3} I_{i} f_{i}(t, u(t))}{\frac{1}{4} t^{2} \sec \left(\frac{\pi t}{3}\right)\left(\frac{|u(t)|+1}{u(t) \mid+2}\right)}\right]=\frac{2}{5} \cos \left(\frac{t}{6}\right)\left(\frac{|u(t)|}{|u(t)|+1}+I^{\frac{5}{2}} u(t)\right), \quad t \in J, \\
u(0)=0, \quad D^{\omega} u(0)=0, \quad u(1)=\delta u(\eta), \quad 0<\delta<1,0<\eta<1,
\end{array}\right.
$$

where

$$
\begin{aligned}
\sum_{i=1}^{3} I^{\beta_{i}} f_{i}(t, u(t))= & I^{\frac{1}{3}}\left(\cos \left(\frac{t}{3}\right)\left(\frac{|u(t)|}{1+|u(t)|}\right)+e^{t} \sin t\right)+I^{\frac{4}{3}}\left(\frac{\sqrt{t} \sin t|u(t)|}{|u(t)|+1}+\frac{2}{3-t^{2}}\right) \\
& +I^{\frac{3}{5}}\left(\frac{|u(t)| \tan \left(\frac{\sqrt{t}}{2}\right)}{|u(t)+2|}+\frac{t}{1+e^{t}}\right) .
\end{aligned}
$$

From equations (16) and (17), we have $\alpha=\frac{3}{5}, \omega=\frac{3}{2}, m=3, \beta_{1}=\frac{1}{3}, \beta_{2}=\frac{4}{3}, \beta_{3}=\frac{3}{5}, \gamma=\frac{5}{2}$, $f_{1}(t, u(t))=\cos \left(\frac{t}{3}\right)\left(\frac{|u(t)|}{1+|u(t)|}\right)+e^{t} \sin t, f_{2}(t, u(t))=\frac{\sqrt{t} \sin t|u(t)|}{|u(t)|+1}+\frac{2}{3-t^{2}}, f_{3}(t, u(t))=\frac{|u(t)| \tan \left(\frac{\sqrt{t}}{2}\right)}{|u(t)+2|}+\frac{t}{1+e^{t}}$, $g(t, u(t))=\frac{1}{4} t^{2} \sec \left(\frac{\pi t}{3}\right)\left(\frac{|u(t)|+1}{|u(t)|+2}\right)$, and $h\left(t, u(t), I^{\gamma} u(t)\right)=\frac{2}{5} \cos \left(\frac{t}{6}\right)\left(\frac{|u(t)|}{|u(t)|+1}+I^{\frac{5}{2}} u(t)\right)$. It is easy to show that

$$
\begin{aligned}
& \mid f_{1}(t, u(t))-f_{1}\left(t, v(t)\left|\leq \cos \left(\frac{t}{3}\right)\right| u(t)-v(t) \mid\right. \\
& \mid f_{2}(t, u(t))-f_{2}(t, v(t)|\leq \sqrt{t} \sin t| u(t)-v(t) \mid \\
& \mid f_{3}(t, u(t))-f_{3}\left(t, v(t)\left|\leq \tan \left(\frac{\sqrt{t}}{2}\right)\right| u(t)-v(t) \mid\right. \\
& \mid g(t, u(t))-g\left(t, v(t)\left|\leq \frac{1}{4} t^{2} \sec \left(\frac{\pi t}{3}\right)\right| u(t)-v(t) \mid\right. \\
& \text { and } \mid h(t, u(t), \bar{u}(t))-h\left(t, v(t), \bar{v}(t) \mid \leq \frac{2}{5} \cos \left(\frac{t}{6}\right)(|u(t)-v(t)|+|\bar{u}(t)-\bar{v}(t)|) .\right.
\end{aligned}
$$


Therefore, we can choose

$$
\begin{aligned}
& \lambda_{1}(t)=\cos \left(\frac{t}{3}\right), \quad \lambda_{2}(t)=\sqrt{t} \sin t, \quad \lambda_{3}(t)=\tan \left(\frac{\sqrt{t}}{2}\right), \\
& \Theta(t)=\frac{1}{4} t^{2} \sec \left(\frac{\pi t}{3}\right), \quad \psi(t)=\frac{2}{5} \cos \left(\frac{t}{6}\right) .
\end{aligned}
$$

Also the functions $g(t, u(t)), h\left(t, u(t), I^{\gamma} u(t)\right)$, and $f_{i}(t, u(t)), i=1,2,3$, are bounded by the positive functions as follows:

$$
\begin{aligned}
& \left|f_{1}(t, u(t))\right| \leq \cos \left(\frac{t}{3}\right)+e^{t} \sin t=\phi_{1}(t), \\
& \left|f_{2}(t, u(t))\right| \leq \sqrt{t} \sin t+\frac{2}{3-t^{2}}=\phi_{2}(t), \\
& \left|f_{3}(t, u(t))\right| \leq \tan \left(\frac{\sqrt{t}}{2}\right)+\frac{t}{1+e^{t}}=\phi_{3}(t), \\
& |g(t, u(t))| \leq \frac{1}{4} t^{2} \sec \left(\frac{\pi t}{3}\right)=\chi(t), \\
& \text { and }|h(t, u(t), v(t))| \leq \frac{2}{5} \cos \left(\frac{t}{6}\right)=\Omega(t)
\end{aligned}
$$

Choosing $\delta=0.1, \eta=0.9$ and putting $\|\Omega\|=\|\psi\|=0.4,\|\chi\|=\|\Theta\|=0.5$ in (8), we have

$$
\left(1+\frac{1+\delta}{|\delta \eta-1|}\right)\left[\frac{\|\Omega\|\|\Theta\|+\|\chi\|\|\psi\|}{\Gamma(\alpha+1) \Gamma(\omega+1)}+\frac{\|\chi\|\|\psi\|}{\Gamma(\alpha+1) \Gamma(\omega+1) \Gamma(\gamma+1)}\right] \approx 0.8594<1 .
$$

Hence all the conditions of Theorem 2 hold. Thus the boundary value problem (16) has at least one solution in $\mathbb{J}=[0,1]$.

\section{Conclusion}

In consequence to generalized Krasnoselskii's fixed point theorem provided by Dhage [1], we developed an existence result for the aforementioned boundary value problem (2) of hybrid fractional sequential integro-differential equations. The respective result has been tested by providing an illustrative example.

\section{Acknowledgements}

We thank the referees for their useful suggestions which improved the final version of the manuscript.

Funding

There is no funding source to support this manuscript financially.

Availability of data and materials

Not applicable.

Competing interests

It is declared that no competing interests exist regarding this manuscript.

Authors' contributions

All authors have equal contribution in this manuscript. All authors read and approved the final manuscript.

\section{Publisher's Note}

Springer Nature remains neutral with regard to jurisdictional claims in published maps and institutional affiliations.

Received: 9 June 2018 Accepted: 7 April 2019 Published online: 18 April 2019 


\section{References}

1. Dhage, B.C., Lakshmikantham, V.: Basic results on hybrid differential equations. Nonlinear Anal. 4, 414-424 (2010)

2. Oldham, K.B., Spanier, J.: The Fractional Calculus. Academic Press, London (1974)

3. Benchohra, B., Graef, J.R., Hamani, S.: Existence results for boundary value problem with nonlinear fractiona differential equation. Appl. Anal. 87, 851-863 (2008)

4. Zhang, S.Q.: The existence of a positive solution for a nonlinear fractional differential equation. J. Math. Anal. Appl. 252, 804-812 (2000)

5. Belarbi, A., Benchohra, M., Ouahab, A.: Existence result for fractional differential equation of fractional order. Appl. Anal. 85, 1459-1470 (2006)

6. Bai, Z., Lu, H.: Positive solutions for boundary value problem of nonlinear fractional differential equation. J. Math. Anal. Appl. 311, 495-505 (2005)

7. Li, C.F., Luo, X.N., Zhou, Y.: Existence of positive solutions of the boundary value problem for nonlinear fractional differential equations. Comput. Math. Appl. 59, 1363-1375 (2010)

8. Babakhani, A., Gejji, V.D.: Existence of positive solutions of nonlinear fractional differential equations. J. Math. Anal. Appl. 278, 434-442 (2003)

9. Zhang, S.Q.: Existence of positive solution for some class of a nonlinear fractional differential equations. J. Math. Anal. Appl. 278, 136-148 (2003)

10. Ahmad, B., Nieto, J.J.: Existence of solutions for anti-periodic boundary value problems involving fractional differential equations via Leray-Schauder degree theory. Topol. Methods Nonlinear Anal. 35, 295-304 (2010)

11. Rehman, M., Khan, R.A.: Existence and uniqueness of solutions for multi-point boundary value problems for fractional differential equations. Appl. Math. Lett. 23, 1038-1044 (2010)

12. Khan, R.A., Rehman, M.: Existence of multiple positive solutions for a general system of fractional differential equations. Commun. Appl. Nonlinear Anal. 18, 25-35 (2011)

13. Khan, R.A., Rehman, M., Asif, N.: Three point boundary value problems for nonlinear fractional differential equations. Acta Math. Sci. 31(B4), 1-10 (2011)

14. Rehman, M., Khan, R.A.: A note on boundary value problems for a coupled system of fractional differential equations. Comput. Math. Appl. 61, 2630-2637 (2011)

15. Benchohra, M., Hamidi, N., Henderson, J.: Fractional differential equations with anti-periodic boundary conditions Numer. Funct. Anal. Optim. 34(4), 404-414 (2013)

16. Wang, J., Li, X.: Ulam-Hyers stability of fractional Langevin equations. Appl. Math. Comput. 258, $72-83$ (2015)

17. Kumama, P., Ali, A., Shah, K., Khan, R.A.: Existence results and Hyers-Ulam stability to a class of nonlinear arbitrary order differential equations. J. Nonlinear Sci. Appl. 10, 2986-2997 (2017)

18. Wang, J., LV, L., Zhou, Y.: Ulam stability and data dependence for fractional differential equations with Caputo derivative. Electron. J. Qual. Theory Differ. Equ. 2011, 63 (2011)

19. Haq, F., Shah, K., Rahman, G., Shahzad, M.: Hyers-Ulam stability to a class of fractional differential equations with boundary conditions. Int. J. Appl. Comput. Math. 3, 1135-1147 (2017)

20. Caputo, M.: Linear models of dissipation whose Q is almost frequency independent. Geophys. J. Int. 13(5), 529-539 (1967)

21. Yang, Y., Ma, Y., Wang, L.: Legendre polynomials operational matrix method for solving fractional partial differential equations with variable coefficients. Math. Probl. Eng. (2015). https://doi.org/10.1155/2015/915195

22. Mainardi, F.: Fractals and Fractional Calculus Continuum Mechanics. Springer, Vienna (1997)

23. Rostamy, D., Karimi, K., Mohamadi, E.: Solving fractional partial differential equations by an efficient new basis. Int. J. Appl. Math. Comput. 5(1), 6-12 (2013)

24. Tarasov, V.E.: Fractional integro differential equations for electromagnetic waves in dielectric media. Theor. Math. Phys. 158(3), 355-359 (2009)

25. Baillie, R.T.: Long memory processes and fractional integration in econometrics. J. Econ. 73, 5-59 (1996)

26. Magin, R.L.: Fractional calculus in bioengineering. Crit. Rev. Biomed. Eng. 32(2), 1-104 (2004)

27. Magin, R.L.: Fractional calculus in bioengineering - part 2. Crit. Rev. Biomed. Eng. 32(2), 105-193 (2004)

28. Magin, R.L.: Fractional calculus in bioengineering-part 3. Crit. Rev. Biomed. Eng. 32(3-4), 194-377 (2004)

29. Hilal, K., Kajouni, A.: Boundary value problem for hybrid differential equations with fractional order. Adv. Differ. Equ. 2015, $183(2015)$

30. Herzallah, M.A.E., Baleanu, D.: On fractional order hybrid differential equations. Abstr. Appl. Anal. 2014, Article ID 389386 (2014). https://doi.org/10.1155/2014/389386

31. Lu, H., Sun, S., Yang, D., Teng, H.: Theory of fractional hybrid differential equations with linear perturbations of second type. Bound. Value Probl. 2013, 23 (2013)

32. Zhao, Y., Sun, S., Han, Z., Li, Q.: Theory of fractional hybrid differential equations. Comput. Math. Appl. 62, 1312-1324 (2011)

33. Ullah, Z., Ali, A., Khan, R.A., Iqbal, M.: Existence results to a class of hybrid fractional differential equations. Matriks Sains Mat. (MSMK) 1, 13-17 (2018)

34. Sitho, S., Ntouyas, S.K., Tariboon, J.: Existence results for hybrid fractional integro-differential equations. Bound. Value Probl. 2015, $113(2015)$

35. Podlubny, I.: Fractional Differential Equation. Academic Press, New York (1999)

36. Kilbas, A.A., Srivastava, H.M., Trujillo, J.J.: Theory and Applications of Fractional Differential Equations. North-Holland Mathematics Studies, vol. 204. Elsevier, Amsterdam (2006)

37. Khan, R.A., Shah, K.: Existence and uniqueness of solutions to fractional order multi-point boundary value problems. Commun. Appl. Anal. 19, 515-526 (2015) 